\section{Normal values of distal radioulnar translation assessed by three-dimensional C-arm scans: a cadaveric study}

\author{
Benedict Swartman ${ }^{1}$, Laura Benner ${ }^{2}$, Stephan Grechenig ${ }^{3}$, \\ Jochen Franke ${ }^{1}$, Paul A. Grützner ${ }^{1}$ and Marc Schnetzke ${ }^{1}$
}

Journal of Hand Surgery

(European Volume) 2019, Vol. 44(5) 503-509 (C) The Author(s) 2019

Article reuse guidelines: sagepub.com/journals-permissions DOI: $10.1177 / 1753193419826486$ journals.sagepub.com/home/jhs

(3)AGE

\begin{abstract}
We investigated whether mobile $\mathrm{C}$-arm cone beam computer tomography (CBCT) could be used to measure radioulnar translation. The study was conducted on 31 Thiel-fixed intact cadaver arms. Three-dimensional scans of each wrist were carried out in pronation and supination. Four established measurement methods were used (radioulnar line, subluxation ratio, epicentre and radioulnar ratio methods) to measure radioulnar translation. The intraclass correlation coefficient for inter-observer and intra-observer reliability were excellent in three of four methods (>0.94). The reference ranges for physiological radioulnar translation were between $-30 \%$ and $91 \%$ (radioulnar line method), $-32 \%$ and $87 \%$ (subluxation ratio method), $-40 \%$ and $23 \%$ (epicentre method), and $2 \%$ and $73 \%$ (radioulnar ratio method). Our results indicate that radioulnar translation in the distal radioulnar joint can be determined reliably using mobile $\mathrm{C}$-arm CBCT. The normal values provide a basis for further experimental and clinical studies.
\end{abstract}

\title{
Keywords
}

Distal radioulnar joint, radioulnar translation, pronosupination, 3-D imaging, normal values, anatomic study

Date received: 10th July 2018; revised: 30th December 2018; accepted: 3rd January 2019

\section{Introduction}

The incidence of accompanying instability of the distal radioulnar joint (DRUJ) after fractures of the distal radius is $10-40 \%$ (Geissler et al., 1996; Kramer et al., 2013; Lindau et al., 2000; Stoffelen et al., 1998; Wijffels and Ring, 2011). Ruptures of the triangular fibrocartilage complex (TFCC), in particular, are associated with DRUJ instability (Lindau et al., 2000). There is no established diagnostic method to determine radioulnar instability. Even in patients with high-grade radioulnar instability such as the Essex-Lopresti injury, the injury to the DRUJ is very often missed (Schnetzke et al., 2017).

The clinical differentiation of an unstable DRUJ from an intact DRUJ remains difficult owing to the low sensitivity, specificity and reproducibility of testing radioulnar translation manually (Pickering et al., 2016; Wijffels et al., 2012). Even in the extreme form of destabilization, rupture of the interosseous membrane of the forearm in Essex-Lopresti injuries, only $70 \%$ of instable cadaveric DRUJ could be detected successfully by the intraoperative clinical examination (Kachooei et al., 2015).

Conventional two-dimensional radiography of the wrist is a static examination and is therefore of limited use in demonstrating DRUJ instability, which varies with pronation and supination. Computed tomography (CT) is better suited to demonstrating instability of the DRUJ, as it allows three-dimensional (3-D) data acquisition of the wrist in pronation and supination (King et al., 1986).

${ }^{1}$ BG-Klinik Ludwigshafen, Department for Orthopedics and Orthopedic Trauma, Trauma Surgery Clinic at Heidelberg University, Ludwigshafen, Germany

${ }^{2}$ Institute of Medical Biometry and Informatics, University of Heidelberg, Heidelberg, Germany

${ }^{3}$ University Hospital Regensburg, Department for Trauma Surgery, Regensburg, Germany

Corresponding Author:

Marc Schnetzke, BG-Klinik Ludwigshafen, Department for Orthopedics and Orthopedic Trauma, Trauma Surgery Clinic at Heidelberg University, Ludwig-Guttmann-Strasse 13, 67071 Ludwigshafen, Germany.

Email: Marc.schnetzkeabgu-ludwigshafen.de 
Radioulnar translation is the relative movement of the two bones with respect to each other. It occurs in the intact DRUJ within a physiological range. Typically, the ulnar head glides anteriorly in the sigmoid notch in supination and moves dorsally in pronation. Four radiological measurement methods based on axial CT reconstructions of the DRUJ have been described for quantifying radioulnar translation (Mino et al., 1983, 1985). These had $80-97 \%$ interand intra-observer agreement (Park and Kim, 2008; Wijffels et al., 2016). Normal values have been determined in the intact DRUJ. However, the examinations were carried out in conscious patients and the range of movement might be altered by pain and muscle tension, which might restrict joint movement.

The aim of this study was to determine physiological normal values for translation in the DRUJ with uninjured anatomical specimens using 3-D imaging with mobile $\mathrm{C}$-arm $\mathrm{CT}$.

\section{Methods}

Thirty-one intact adult cadaver upper limbs Imean age at the time of death 74 years; range 68-84 years) which had been donated to the Department of Anatomy of the Medical University of Graz for scientific research under the approval of the Anatomical Donation Program of the University of Graz were used. In each case, complete arms including the shoulder joints were available. The arms were not paired. The specimens were fixed according to Thiel's method and were thus realistic in consistency, elasticity and colour (Thiel, 1992; Vollner et al., 2017). The arms were examined macroscopically and radiologically to ensure they were in an undamaged condition without signs of previous injuries.

The arms were positioned in a specially designed retention device that allowed flexion of the elbow at $90^{\circ}$ and maximum rotation of the forearm in pronation and supination (Figure 1). The limbs were positioned in their maximum range of pronation and supination. Angles were measured by a goniometer (Kirchner und Wilhelm, Asperg, Germany) and the means were calculated. The mean maximum range of motion of the restrained limbs was $89^{\circ}$ (SD 2) pronation and $86^{\circ}$ (SD 4) supination.

The position of the lower arm (pronation or supination) was secured with a strapped-on bar (Figure 1). A 3-D scan of the wrist was performed in each position using a 3-D-capable image converter (Arcadis Orbic, Siemens Healthcare GmbH, Erlangen, Germany). The reconstructions could be freely selected so that axial layers at $90^{\circ}$ to the axis of the radial shaft and corresponding to those of Park and Kim (2008) and Wijffels et al. (2016) were generated. In the axial plane, the sigmoid notch grows wider or narrower on scrolling through the layers due to the shape of the distal radius. The region of interest is the widest part of the notch based on the axial planes. In order to realize comparable planes in all datasets, the following landmarks had to be apparent: the widest part of the sigmoid notch; the ulnar head; and Lister's tubercle (Figure 2). The images were printed on paper at $1.5 \times$ magnification to

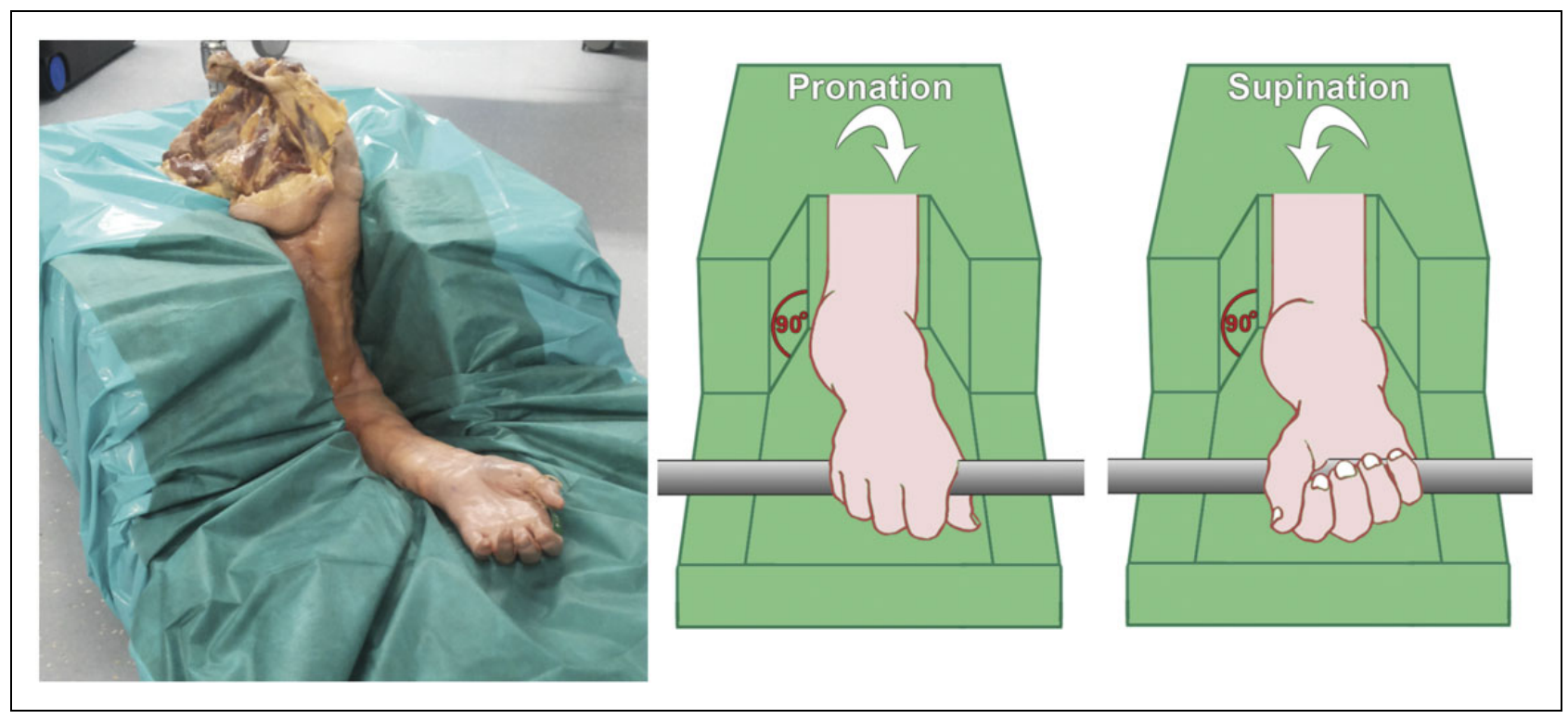

Figure 1. The anatomical specimens were positioned in a retaining device with the elbow in $90^{\circ}$ flexion. The lower arms were pronated and supinated at $90^{\circ}$ by being connected to a rod. 


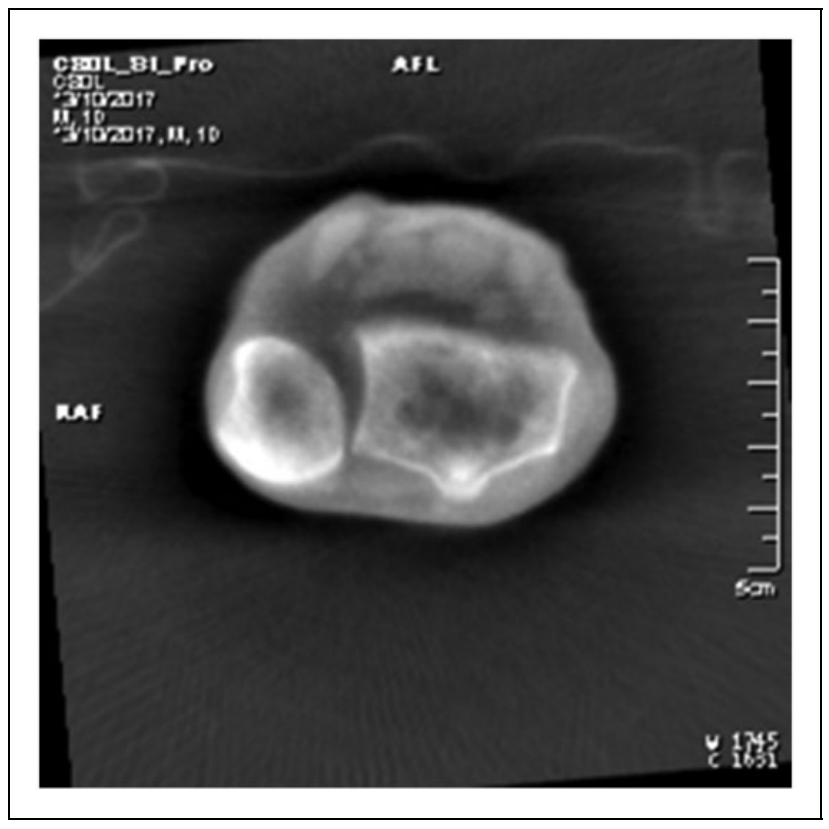

Figure 2. Axial reconstruction of the wrist at the level of Lister's tubercle and the ulnar head, based on the 3-D dataset from $\mathrm{C}$-arm CT. The side pointing upwards in the image depicts the anterior aspect of the distal radius. The wrist in this view is in $90^{\circ}$ pronation.

allow manual measurement. The specific methods are described further on: in all cases the lines were drawn by waterproof pen, angles and distances were measured by a set square and the ratios calculated electronically. The measurement methods used were the radioulnar line $(R L)$ method, subluxation ratio (SR) method, epicentre (EC) method and radioulnar ratio (RR) method (Figure 3). All four techniques were used to identify the one(s) with the best reliability. All the methods quantify the position of the ulnar head relative to the sigmoid notch of the distal radius and give a length ratio as a percentage. Dorsal displacements are documented with a plus sign and anterior displacements with a minus sign. In case the ulnar head exceeded both lines, dorsal and anterior, both distances were measured and added together. Four measurements were made on each view (pronation and supination). The 248 measurements were carried out in random sequence by two blinded examiners (B.S. and M.S.). For the intra-rater reliability, examiner 1 (B.S.) repeated his measurements after $>3$ months.

The plotted lines and distances are shown in Figure 3. With the RL method (Figure 3a), the anterior $(A-C)$ and the dorsal (B-D) radial and ulnar limit of the radius were each joined by a line. The maximum distance of the ulnar head outside the intervening area was measured perpendicularly to the nearest line $(E-F)$. If the ulnar head lay between the two lines, the result was 0 . These distances were expressed as a ratio of the length of the sigmoid notch: $(E-F) /(A-B)$. With the subluxation ratio $(S R)$ method (Figure $3 b$ ), the two edges of the sigmoid notch were joined by a line $(A-B)$ and a perpendicular intersecting line was drawn through it at each of the two endpoints. The distance of the ulnar head was measured perpendicularly to the nearest line (C-D); the ratio $(C-D) /(A-B)$ was determined similarly. For the measurement by the epicentre method (EC) (Figure $3 \mathrm{c}$ ), a joining line was also drawn between the two edges of the sigmoid notch (A-B) and half this distance marked as ' $C$ '. The epicentre of the ulna was defined as half the distance between the centre of the ulnar head (E) and the centre of the ulnar styloid process (D) and called ' $F$ '. The centres were determined using a template with concentric circles along the bony outer edges or the ulnar joint area. The connecting line of point ' $F$ ' running perpendicularly to $A B$ marks a point $(G)$ at the intersection with $A B$. The distance $C-G$ is expressed as a ratio to the distance $A-B$. For the determination by the RR method (Figure 3d), again a line was drawn between the anterior and dorsal limits of the sigmoid notch (A-B). A second line at right angles to the previous one was drawn through the centre of the ulnar head $(C)$ and marked point $D$ at its intersection with $A B$. The ratio between $A-D$ and $A-B$ was determined.

Each measuring method generates a ratio between the position of the ulna relative to the length of the corresponding sigmoid notch. This ratio changes between pronated and supinated wrists according to the radioulnar translation. Larger differences therefore represent larger translation distances. The numerical values obtained by each method can vary from those obtained using other methods on the same image.

To reflect the actual translation of the ulnar head relative to the sigmoid notch of the distal radius, the differences between pronation and supination were determined for each specimen:

$$
\Delta_{s}=\operatorname{Pro}_{s}-\operatorname{Sup}_{s}
$$

( $\Delta=$ radioulnar translation; Pro $=$ ratio in pronation; Sup $=$ ratio in supination; $s=$ specimen number)

The mean and $95 \%$ confidence interval of the differences for each of the four measurement methods were calculated to determine their distribution within the 31 specimens. The mean of the four techniques themselves could not directly be compared with each other, since they produce different values due to their individual measuring methods.

In order to define the 'normal ranges' of radioulnar translation in the intact specimen, the equivalent 


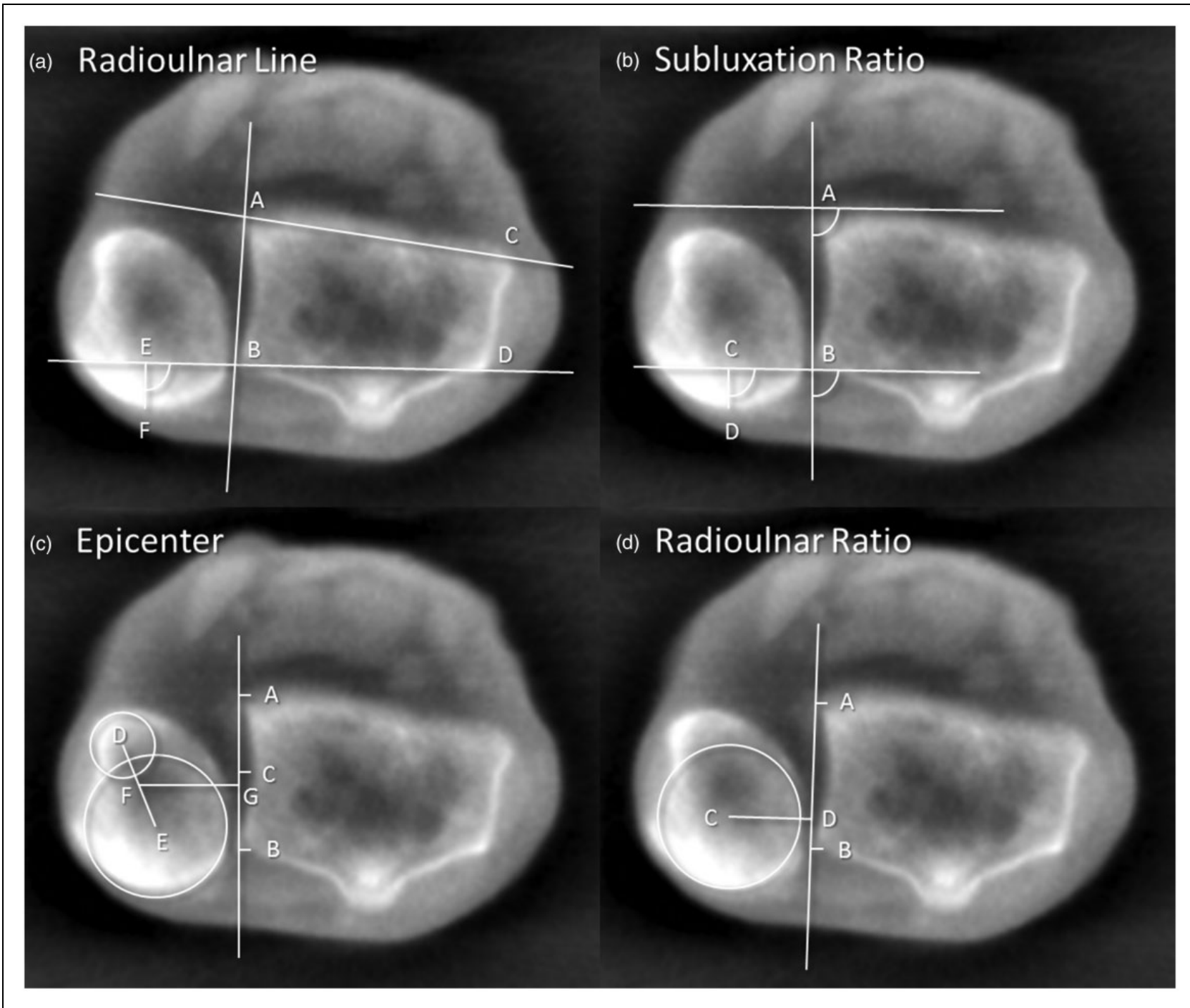

Figure 3. Depiction of the four measurement methods using axial reconstructions of the 3-D dataset from the mobile $C$ arm. The methods are based on the sigmoid notch of the distal radius and on the ventral and dorsal radial edges (a, b) or on the centre of rotation of the ulnar head $(c, d)$.

'95\% reference ranges' were calculated, similar to Wijffels et al. (2016), using the following formula:

$$
R_{m}=\text { mean }_{m} \pm 1.96 \times \text { standard deviation }_{m}
$$

( $\mathrm{R}=$ reference range; mean/standard deviation $=$ mean/standard deviation of 31 specimens; $m=$ measuring method)

All values within these reference ranges were taken as the normal values.

Interrater and intrarater reliabilities were determined with the intraclass correlation coefficient (ICC) (model 2,1) (Landis and Koch, 1977). ICCs $<0.40$ indicate poor agreement, $0.40-0.59$ moderate agreement, $0.60-0.74$ good agreement, $0.75-0.89$ very good agreement and $\geq 0.90$ excellent agreement.

\section{Results}

With the RL method, the mean ratios within the 31 specimen were $16 \%$ (SD 19) for pronation and $-14 \%$ (SD 17) for supination; with the subluxation ratio method, 19\% (SD 19) for pronation and $-8 \%$ (SD 19) for supination; with the epicentre method, 30\% (SD 24) for pronation and 38\% (SD 26) for supination; and with the RR method, $71 \%$ (SD 10) for pronation and $34 \%$ (SD 11) for supination.

The mean differences between pronation and supination as a value for the radioulnar translation 
and the $95 \%$ confidence intervals and $95 \%$ reference ranges are shown in Table 1. The histograms with the cut-off values of the $95 \%$ reference ranges are depicted in Figure 4. The distribution of the

Table 1. Normal values of the different measuring methods (in percentages), each based on 31 specimens. The 95\% reference interval is defined as the normal range of radioulnar translation of the stable distal radioulnar joint.

\begin{tabular}{lll}
\hline & $\begin{array}{l}\text { Mean (SD) } \\
\text { (95\% Cl) }\end{array}$ & $\begin{array}{l}\text { 95\% reference } \\
\text { interval } \\
\text { (normal range) }\end{array}$ \\
\hline Radioulnar line & $31(31)(20$ to 41$)$ & -30 to 91 \\
Subluxation ratio & $28(30)(17$ to 38$)$ & -32 to 87 \\
Epicentre & $-8(16)(-14$ to -3$)$ & -40 to 23 \\
Radioulnar ratio & $37(18)(31$ to 44$)$ & 2 to 73 \\
\hline
\end{tabular}

SD: standard deviation, $\mathrm{Cl}$ : confidence interval. measurement values within the individual measurement methods were deemed to be normally distributed.

The ICCs for interrater reliability were: RL method =ICC 0.96 (95\% Cl: 0.91 to 0.99$)$ (excellent); subluxation ratio method $=$ ICC $0.98(95 \% \mathrm{Cl}: 0.94$ to 0.99) (excellent); epicentre method =ICC $0.71 \quad 195 \%$ $\mathrm{Cl}: 0.39$ to 0.87 ) (good); and RR method $=$ ICC 0.94 (95\% Cl: 0.85 to 0.98) (excellent). The values for intrarater reliability were: $\mathrm{RL}$ method $=\mathrm{ICC} 0.94(95 \% \mathrm{Cl}: 0.88$ to 0.97 ) (excellent); subluxation ratio method $=$ ICC 0.96 (95\% Cl: 0.93 to 0.98 ) (excellent); epicentre method= ICC 0.89 (95\% Cl: 0.77 to 0.94$)$ (very good); and RR method = ICC 0.95 (95\% Cl: 0.89 to 0.97) (excellent).

\section{Discussion}

In our study, we were able to show that radioulnar translation in the DRUJ could reliably be determined

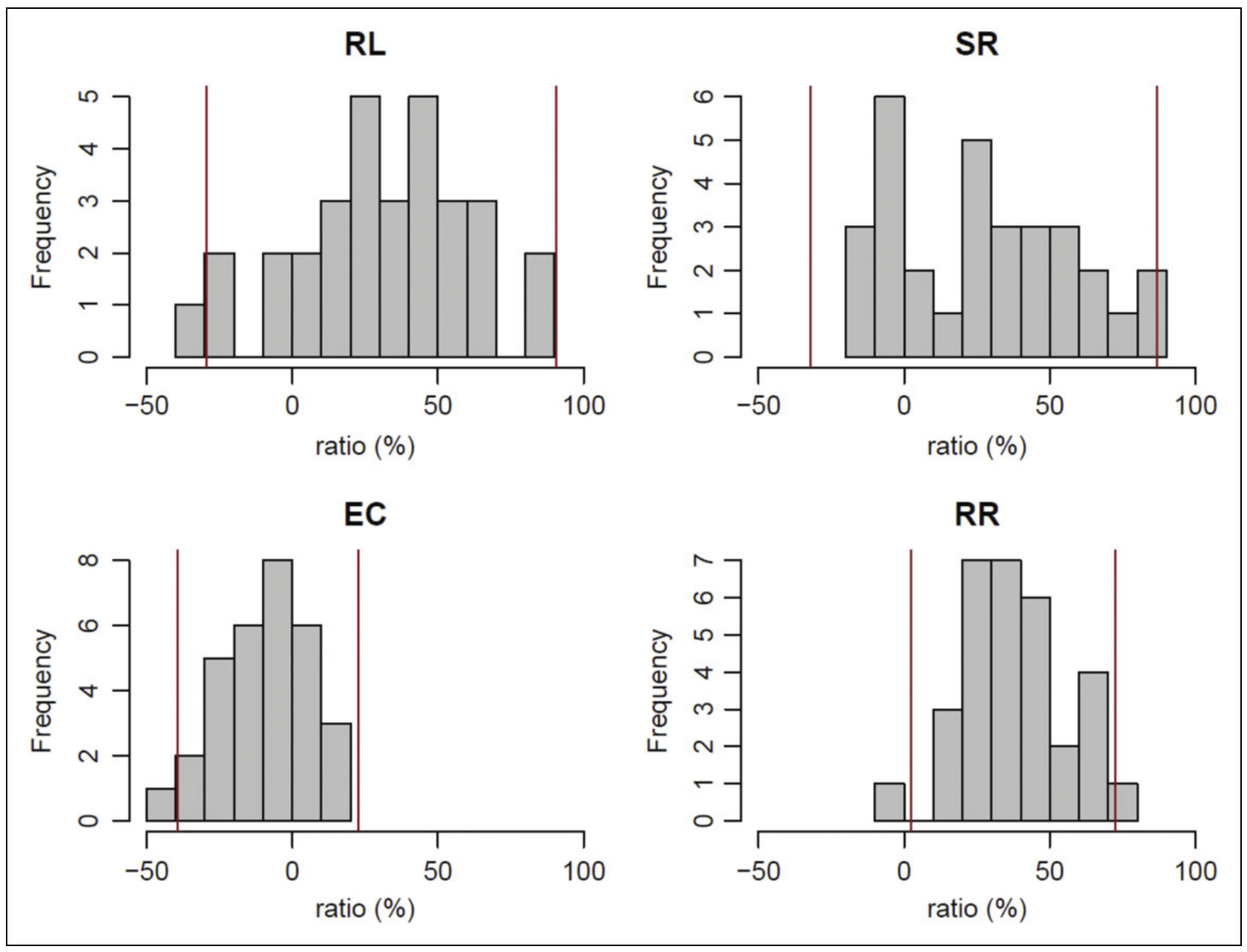

Figure 4. The histograms represent the statistical distributions of the mean differences between pronation and supination, showing radioulnar translation in the DRUJs of 31 patients within the different measurement techniques. Normal distribution can be assumed from the bell-shaped plots. Vertical lines mark the 95\% reference interval. RL: radioulnar line method; SR: subluxation ratio method; EC: epicentre method; RR: radioulnar ratio method. 
by mobile $\mathrm{C}$-arm cone beam CT. Except for the epicentre method, all measurement methods showed excellent inter-observer and intra-observer reliabilities. The normal values of radioulnar translation that we report can provide a basis for further experimental and clinical studies.

Ranges of normal values for radioulnar translation in the DRUJ have previously been described (Park and Kim, 2008; Wijffels et al., 2016). However, these were determined by CT in conscious patients. With a view to subsequent intraoperative use, we undertook measurements using 3-D-capable mobile C-arm CT. We used a cadaveric study, as in this case muscle tone is reduced. Though not representative of a patient under general anaesthesia, the realistic structure and elasticity of the soft tissues in Thielfixed specimens have been confirmed in previous studies (Thiel, 1992; Vollner et al., 2017).

The inter-observer and intra-observer reliability in our study differ somewhat from those reported by others. In the study by Wijffels at al. (2016), the epicentre method showed the highest inter-observer agreement with 0.73 and the highest intra-observer agreement with 0.82 . In our study, the epicentre method came out worst with an inter-observer agreement of 0.71 and an intra-observer agreement of 0.885 . This discrepancy can be explained by the different study designs. One main difference is the fact that the CT planes are not freely adjustable in contrast to the $\mathrm{C}$-arm. In the previous studies, the patients held on actively to a bar, which automatically recruits the secondary stabilizers of the DRUJ. Up to now the measurement of radioulnar translation has been based on CT imaging (King et al., 1986). However, we see some benefits in diagnostic investigations using a mobile C-arm. Diagnosing DRUJ instability may have immediate operative consequences, for example, the surgeon may decide to stabilize the DRUJ acutely. Mikic (1995) and Schnetzke et al. (2017) showed that patients benefit from early Kirschner wire transfixation of the DRUJ. In Galeazzi and Essex-Lopresti injuries in particular, DRUJ instability may be assumed. Mobile 3-D C-arms are fairly widely available. Unlike a static CT scan, with the mobile $\mathrm{C}$-arm cone beam procedure, it is possible for the examiner or operator to process and set the relevant planes in advance. The normal data of radioulnar translation have previously been given separately for the maximum rotation of the lower arm in pronation and supination (Park and Kim, 2008; Wijffels et al., 2016). This makes interpretation laborious. For this reason, we calculated the difference between pronation and supination for each specimen and measurement method and as a result expect a simplified numerical presentation of the
Table 2. Comparison of reference intervals between two methods of measurement (in percentages).

\begin{tabular}{lrl}
\hline & \multicolumn{2}{c}{$95 \%$ reference interval (normal range) } \\
\cline { 2 - 3 } & Mobile C-arm scan & Fixed CT scan* \\
\hline Radioulnar line & -30 to 91 & -23 to 82 \\
Subluxation ratio & -32 to 87 & -29 to 73 \\
Epicentre & -40 to 23 & -54 to 17 \\
Radioulnar ratio & 2 to 73 & -21 to 68
\end{tabular}

*Data from Wijffels M et al., Skeletal Radiol. 2016, 45: 1487-93.

actual translation. The normal ranges land $95 \%$ reference intervals) of the four measurement methods were given by Wijffels et al. (2016) for both pronation and supination. If these are subtracted from one another and the minimum and maximum possible differences are calculated, this yields values that match our results (Table 2). The only striking finding is the difference in the minimum values of the RR method. This is possibly best explained by the fact that only positive values can occur in measurements made using this method, provided that the ulnar head is not completely luxated from the sigmoid notch in the volar direction. Thus Wijffels et al. (2016) also predominantly obtained positive values with this method, with high values being found for supination, which accordingly resulted in high negative values when the difference is calculated.

This study is limited by the cadaver set-up and the fact that the model lacked the input of dynamic stabilizers. In addition, there has been controversy over whether there is a potential for mechanical degradation of ligaments due to the Thiel embalming method. However, Völlner et al. (2017) reported that the stiffness of the medial and lateral ligament complexes in the knee was similar in cadavers embalmed with the Thiel method and in living patients undergoing total knee arthroplasty. They concluded that Thiel fixation seems to preserve the soft-tissue properties so that they are similar to those in vivo. Owing to the age of the specimens, extrapolation to the general population is only possible to a limited extent. Furthermore, the number of measurements was small, although similar to the numbers in previous reports (Park and Kim, 2008; Wijffels et al., 2016).

Despite these limitations, we consider that it is possible to define reference ranges of radioulnar translation in the DRUJ which will serve as a basis for further experimental and clinical studies.

Acknowledgements Prof. Friedrich Anderhuber, Macroscopic and Clinical Anatomy, Medical University of Graz, Austria, provided the anatomical specimens. 
Declaration of conflicting interests The authors declared the following potential conflicts of interest with respect to the research, authorship, and/or publication of this article: J. Franke, S.Y. Vetter and P.A. Grützner are paid lecturers for Siemens Healthcare $\mathrm{GmbH}$ (Erlangen, Germany).

Funding The authors disclosed receipt of the following financial support for the research, authorship, and/or publication of this article: This work was supported by AOTrauma, Germany (AO Start-up Grant S-16-99S).

Ethical approval Obtained from Rhineland-Palatinate Local Ethics Committee, 55019 Mainz, Germany (No. 837.299.17 (11133)).

\section{References}

Geissler WB, Fernandez DL, Lamey DM. Distal radioulnar joint injuries associated with fractures of the distal radius. Clin Orthop Relat Res. 1996, 327: 135-46.

Kachooei AR, Rivlin M, Wu F, Faghfouri A, Eberlin KR, Ring D. Intraoperative physical examination for diagnosis of interosseous ligament rupture-cadaveric study. J Hand Surg Am. 2015, 40: 1785-90.

King GJ, McMurtry RY, Rubenstein JD, Ogston NG. Computerized tomography of the distal radioulnar joint: Correlation with ligamentous pathology in a cadaveric model. J Hand Surg Am. 1986, 11: 711-7.

Kramer S, Meyer H, O'Loughlin PF, Vaske B, Krettek C, Gaulke R. The incidence of ulnocarpal complaints after distal radial fracture in relation to the fracture of the ulnar styloid. J Hand Surg Eur. 2013, 38: 710-7.

Landis JR, Koch GG. An application of hierarchical kappa-type statistics in the assessment of majority agreement among multiple observers. Biometrics. 1977, 33: 363-74.

Lindau T, Adlercreutz C, Aspenberg P. Peripheral tears of the triangular fibrocartilage complex cause distal radioulnar joint instability after distal radial fractures. J Hand Surg Am. 2000, 25: 464-8.
Mikic ZD. Treatment of acute injuries of the triangular fibrocartilage complex associated with distal radioulnar joint instability. J Hand Surg Am. 1995, 20: 319-23.

Mino DE, Palmer AK, Levinsohn EM. The role of radiography and computerized tomography in the diagnosis of subluxation and dislocation of the distal radioulnar joint. J Hand Surg Am. 1983, 8: 23-31.

Mino DE, Palmer AK, Levinsohn EM. Radiography and computerized tomography in the diagnosis of incongruity of the distal radio-ulnar joint. A prospective study. J Bone Joint Surg Am. 1985, 67: 247-52.

Park MJ, Kim JP. Reliability and normal values of various computed tomography methods for quantifying distal radioulnar joint translation. J Bone Joint Surg Am. 2008, 90 : 145-53.

Pickering GT, Nagata H, Giddins GE. In-vivo three-dimensional measurement of distal radioulnar joint translation in normal and clinically unstable populations. J Hand Surg Eur. 2016, 41: 521-6.

Schnetzke M, Porschke F, Hoppe K, Studier-Fischer S, Gruetzner PA, Guehring T. Outcome of early and late diagnosed Essex-Lopresti injury. J Bone Joint Surg Am. 2017, 99: 1043-50.

Stoffelen D, De Smet L, Broos P. The importance of the distal radioulnar joint in distal radial fractures. J Hand Surg Br. 1998, 23: 507-11.

Thiel W. [The preservation of the whole corpse with natural color]. Ann Anat. 1992, 174: 185-95.

Vollner F, Pilsl U, Craiovan B et al. Stability of knee ligament complex of thiel-embalmed cadaver compared to in vivo knee. J Mech Behav Biomed Mater. 2017, 71: 392-6.

Wijffels M, Brink P, Schipper I. Clinical and non-clinical aspects of distal radioulnar joint instability. Open Orthop J. 2012, 6: 204-10.

Wijffels M, Ring D. The influence of non-union of the ulnar styloid on pain, wrist function and instability after distal radius fracture. J Hand Microsurg. 2011, 3: 11-4.

Wijffels M, Stomp W, Krijnen P, Reijnierse M, Schipper I. Computed tomography for the detection of distal radioulnar joint instability: Normal variation and reliability of four CT scoring systems in 46 patients. Skeletal Radiol. 2016, 45: 1487-93. 